\title{
ON PROPERTY (FA) FOR WREATH PRODUCTS
}

\author{
YVES CORNULIER AND ADITI KAR
}

\begin{abstract}
We characterize permutational wreath products with Property (FA). For instance, the standard wreath product $A$ ? $B$ of two nontrivial countable groups $A, B$, has Property (FA) if and only if $B$ has Property (FA) and $A$ is a finitely generated group with finite abelianisation. We also prove an analogous result for hereditary Property (FA). On the other hand, we prove that many wreath product with hereditary Property (FA) are not quotients of finitely presented groups with the same property.
\end{abstract}

\section{INTRODUCTION}

Property (FA) was introduced by Serre in his monograph [16]: a group $G$ is said to have Property (FA) if every isometric action of $G$ on a (simplicial) tree has a fixed point. Serre's fundamental result [16, Theorem I.6.15] about Property (FA) says that a group $G$ has Property (FA) if and only if the three following conditions are satisfied

- $G$ is not a nontrivial amalgam;

- $G$ has no quotient isomorphic to $\mathbf{Z}$;

- $G$ is not the union of a properly increasing sequence of subgroups.

If $G$ is denumerable, the last condition is equivalent to the requirement that $G$ is finitely generated. In general, it is referred to in the literature as " $G$ has cofinality $\neq \omega$ " and is fulfilled by some uncountable groups [14]. Traditional examples of finitely generated groups with Property (FA) include

(1) finitely generated torsion groups;

(2) Coxeter groups defined by a Coxeter matrix with no occurrence of $\infty$;

(3) special linear groups over the integers, $\mathrm{SL}_{n}(\mathbf{Z})$, for $n \geq 3$;

(4) more generally, groups with Kazhdan's Property (T);

Date: April 15, 2010.

2000 Mathematics Subject Classification. Primary 20E22; Secondary 20E08, 20E06, 20F05.

The second author is supported by EPSRC grant EP/F031947/1. 
(5) irreducible lattices in semisimple Lie groups of real rank at least two, e.g. $\mathrm{SL}_{2}(\mathbf{Z}[\sqrt{2}])$.

The first three of these examples were explained by Serre in [16]; (41) was proved by Watatani in 21], using the characterisation of property (T) in terms of affine actions on Hilbert spaces; and finally (5) was proved by Margulis (see [15]).

The aim of this article is to investigate Property (FA) for wreath products. We recall that given two groups $A, B$ and a $B$-set $X$, their (permutational) wreath product is defined as the group

$$
A \imath_{X} B:=A^{(X)} \rtimes B
$$

where

$$
A^{(X)}=\bigoplus_{x \in X} A_{x}
$$

is the direct sum of isomorphic copies $A_{x}$ of $A$ indexed by $X$. In the special case when $X=B$ with $B$ acting by left multiplication on itself, one obtains the standard wreath product and this, we denote simply as $A<B$. If $A$ and $B$ are finitely generated and $X$ has finitely many $B$-orbits, then $A \tau_{X} B$ is finitely generated as well.

Theorem 1.1. Consider the permutational wreath product $G=A \imath_{X} B$. Assume that $A \neq\{1\}, X \neq \emptyset$ and $X$ has finitely many $B$-orbits, each of which contains more than one element. The following are equivalent

- G has Property (FA);

- B has Property (FA) and $A$ is a group with finite abelianisation, which cannot be expressed as the union of a properly increasing sequence of subgroups.

Contrast with the following result on property (T) groups [7, Proposition 2.8.2]: the wreath product $A$ < $B$ of two non-trivial groups $A, B$ has property (T) if and only if $A$ has property (T) and $B$ is finite.

The following is a well-known problem (it appears for instance as 4 . Question 7] and in [19]).

Question 1.2 (fg versus fp). Is every finitely generated group with Property (FA) the quotient of a finitely presented group with prop$\operatorname{erty}(\mathrm{FA})$ ?

It can also be stated as "is Property (FA) open in the space of marked groups?" (see [8, Section 2.6(h)]). The analogous question for some other fixed point properties has a positive answer

- for Property (FR) (fixed point property on $\mathbf{R}$-trees), a result of Culler and Morgan [9, Proposition 4.1]. 
- Property (FH) (fixed point property on Hilbert spaces, also known as Kazhdan's Property (T)), a result independently due to Shalom and Gromov ([18, Theorem 6.7] and [11, 3.8.B])

- more generally, again by Gromov [11, 3.8.B], the fixed point property on any class of metric spaces which is stable under "scaling ultralimits", e.g. the class of all CAT(0)-spaces.

It is an old open question [17, Question A, p.286] whether Property (FA) implies the a priori stronger Property (FR). Of course a positive answer would imply a positive answer to Question 1.2.

Some evidence for a positive answer for Question 1.2 is given by the case of wreath products. From the proof of Theorem 1.1, one can deduce the proposition below.

Proposition 1.3. Let $A$ and $B$ be finitely presented groups and let $X$ be a $B$-set with finitely many orbits. If in addition, $A$ has finite abelianisation and $B$ has Property (FA), then $A l_{X} B$ is the quotient of a finitely presented group with Property (FA).

Note that Baumslag [2] proved that a wreath product of non-trivial finitely presented groups $A$ \& $B$ is finitely presented only when $B$ is finite.

Definition 1.4. A group $G$ has hereditary Property (FA) if $G$ and all its finite index subgroups have Property (FA).

It is natural to address Question 1.2 with Property (FA) replaced by hereditary (FA). In this situation, the answer turns out to be negative and wreath products provide a large class of elementary examples.

Theorem 1.5. Let $G=A$ \& $B$ be the standard wreath product of two finitely generated groups. Assume that $B$ is an infinite, residually finite group and that $A$ has at least one non-trivial finite quotient. Then every finitely presented group mapping onto $G$ has a finite index subgroup with a surjective homomorphism onto a non-abelian free group.

The next theorem, which relies on Theorem 1.1 and further arguments, shows how to chose the group $G$ from Theorem 1.5 to have hereditary (FA).

Theorem 1.6. Let $G=A$ \& $B$ be a wreath product of finitely generated groups, with $B$ infinite. The following are equivalent

- $G$ has hereditary Property (FA);

- B has hereditary Property (FA) and A has finite abelianisation.

Example 1.7. If $G=F$ 2 $\mathrm{SL}_{3}(\mathbf{Z})$ with $F$ any non-trivial finite group, then $G$ has hereditary Property (FA) by Theorem 1.6, but is not the 
quotient of any finitely presented group with the same property, by Theorem 1.5 .

Remark 1.8. Despite the analogy between Theorems 1.1 and 1.6, Theorem 1.5 shows that Proposition 1.3 is false when (FA) is replaced by hereditary (FA).

Remark 1.9. Theorems 1.5 and 1.6 provide many instances (illustrated by Example 1.7) of groups with hereditary Property (FA), which are not quotients of finitely presented groups with hereditary Property (FA). Here is another one, of a different kind. Let $\Gamma$ be the first Grigorchuk group [13, Chap. VIII]. This is a finitely generated group every proper quotient of which is finite; in particular it cannot be expressed as a non-trivial wreath product with an infinite quotient. Also, it is a finitely generated torsion group and therefore has hereditary Property (FA). It follows however from [10] (see also [3, Corollary 8]) that every finitely presented group mapping onto $\Gamma$ has a finite index subgroup mapping onto the free group.

Remark 1.10. Theorem 1.1 holds when Property (FA) replaced by (FR), with a similar proof.

Remark 1.11. It is not hard to extend Theorem 1.6 to permutational wreath products. On the other hand, the extension of Theorem 1.5 to permutational wreath products is more delicate.

Acknowledgements. We wish to thank Nikolay Nikolov, Armando Martino, Ashot Minasyan and Joël Riou for valuable discussions and suggestions; we are grateful to Indira Chatterji, Luc Guyot and Alain Valette for reading the manuscript and their comments.

\section{Property (FA)}

In this part, we prove Theorem 1.1 and Proposition 1.3. If a group $G$ acts on a set $X$, we denote by $X^{G}$ the set of $G$-fixed points in $X$. We think of each tree as the set of its own vertices. We have the two following standard lemmas.

Lemma 2.1. Suppose that a group $H$ acts on a tree $T$ without inversions. Let $A$ and $B$ be subgroups of $H$ such that $T^{A}$ and $T^{B}$ are non-empty. If $[A, B]=1$ then $T^{A} \cap T^{B} \neq \emptyset$.

Proof. On the contrary, suppose that $T^{A} \cap T^{B}$ is empty. Then, there is a unique geodesic segment $\alpha$ in $T$, of minimal length, joining $T^{A}$ and $T^{B}$. However, as $A$ and $B$ commute, $A$ preserves the set $T^{B}$. This implies that $A$ pointwise fixes the geodesic segment $\alpha$, hence fixes at 
least one element of $T^{B}$, which contradicts the assumption that $T^{A} \cap T^{B}$ is empty.

We say that an action of a group $G$ on a tree $T$ is parabolic if every element of $G$ has a fixed point, but there is no global fixed point.

Lemma 2.2. If $G$ has a parabolic action on a tree, then $G$ is the union of a properly increasing sequence of subgroups.

Proof. By a classical theorem of Tits [20, Proposition 3.4], there exists an end of $T$ which is strongly fixed by $G$. In other words, there exists a geodesic ray $\left(v_{n}\right)$ such that for every $g \in G, g \cdot v_{n}=v_{n}$ for $n$ large enough. Define the non-decreasing sequence of stabilizers

$$
G_{n}=\left\{g \in G: g \cdot v_{k}=v_{k}, \forall k \geq n\right\} .
$$

By assumption, $G=\bigcup G_{n}$. But $G \neq G_{n}$ because $G$ has no global fixed point.

The main idea of the proof of Theorem 1.1 is given by the following result.

Proposition 2.3. Let $A$ and $B$ be groups. Let $X$ be a $B$-set with $X^{B}=\emptyset$, and write $W=A^{(X)}$. Suppose that the permutational wreath product $G=A \imath_{X} B=W \rtimes B$ acts without inversions on a tree $T$. Assume that $T^{B} \neq \emptyset, T^{W}=\emptyset$, and that for any $x$, the action of $A_{x}$ on $T$ is not parabolic. Then there exists a unique geodesic line $\mathcal{L} \subset T$ preserved by $W$. Moreover, the $W$-action on $\mathcal{L}$ is non-trivial and by translations.

Proof. Let us first prove the proposition with the extra-assumption that $G$ acts transitively on $X$, and we fix a basepoint $o$ in $X$.

- First, we prove that $T^{A_{o}}=\emptyset$. Assume the contrary. For each $b \in B$, we have $b . T^{A_{o}}=T^{A_{b . o}}$; in particular, by transitivity, the subtree $T^{A_{x}}$ is nonempty for each $x \in X$. Moreover, for $x \neq o, A_{o}$ and $A_{x}$ commute. Therefore, by Lemma 2.1, we obtain $T^{A_{o}} \cap T^{A_{x}} \neq \emptyset$.

By transitivity of the $G$-action on $X$, we see that $G$ is generated by $A_{x}$ and $B$ for any $x \in X$. Accordingly, if for some $x$, $T^{A_{x}} \cap T^{B} \neq \emptyset$, then we deduce that $T^{G} \neq \emptyset$ and in particular $T^{W} \neq \emptyset$, a contradiction.

Therefore, $T^{A_{x}} \cap T^{B}=\emptyset$ for any $x \in X$. Denote by $u^{x}$ the unique vertex in $T^{A_{x}}$ closest to $T^{B}$. Note that we have $u^{b . x}=b . u^{x}$ for all $b \in B$. For any vertex $v$ in $T$, denote by $\left(v_{0}, v_{1}, \ldots, v_{\ell(v)}=v\right)$ the geodesic segment joining $T^{B}$ to $v$ (so 
$\left.\ell(v)=d\left(v, T^{B}\right)\right)$. Since $T$ is a tree, if for some $x \in X, v$ belongs to the subtree $T^{A_{x}}$ and $k_{x}=d\left(T^{B}, T^{A_{x}}\right)$, we have $u^{x}=v_{k_{x}}$. Now

$$
\begin{aligned}
k_{b . x} & =d\left(T^{B}, T^{A_{b . x}}\right)=d\left(b . T^{B}, b . T^{A_{x}}\right) \\
& =d\left(T^{B}, T^{A_{x}}\right)=k_{x} .
\end{aligned}
$$

Picking $v$ in $T^{A_{o}} \cap T^{A_{b . o}}$, we deduce that $u^{b . o}=v_{k_{b . o}}=v_{k_{o}}=u^{o}$; thus $u^{b . o}=u^{o}$ for all $b$. So $u^{o}$ is invariant under $B$, hence $T^{A_{o}} \cap T^{B} \neq \emptyset$, a contradiction.

- Now we know that $T^{A_{o}}=\emptyset$. If every element of $A_{o}$ fixes some element of $T$, the action of $A_{o}$ on $T$ is, by definition, parabolic; this is ruled out by hypothesis. Accordingly, $A_{o}$ contains an element $a$ acting hyperbolically on $T$. Pick $b \in B$ with $b . o \neq o$; then $a^{\prime}=b a b^{-1}$ also acts hyperbolically on $T$. Let $\mathcal{L}, \mathcal{L}^{\prime}$ denote the axes of $a$ and $a^{\prime}$. For any $x \in X-\{o\}$ (resp. $\in X-$ $\{b . o\}), A_{x}$ centralises $a\left(\right.$ resp. $\left.a^{\prime}\right)$, so $A_{x}$ preserves $\mathcal{L}$ (resp. $\mathcal{L}^{\prime}$ ). In particular, $a^{\prime}$ preserves $\mathcal{L}$, but since $\mathcal{L}^{\prime}$ is the unique axis preserved by $a^{\prime}$, we deduce that $\mathcal{L}=\mathcal{L}^{\prime}$. Therefore, for any $x \in X, A_{x}$ preserves $\mathcal{L}$. Now we claim that $A_{o}$ preserves the orientation of $\mathcal{L}$; otherwise, it contains some element $a^{\prime \prime}$ having a unique fixed point on $\mathcal{L}$. Since $a^{\prime}$ centralizes $a^{\prime \prime}$, we deduce that this point is also fixed by $a^{\prime}$, a contradiction since $a^{\prime}$ acts by non-trivial translation on $\mathcal{L}$. By conjugating, we deduce that for each $x, A_{x}$ preserves the orientation of $\mathcal{L}$, so the whole action of $W$ on $\mathcal{L}$ is by translations and non-trivial.

Finally we have to tackle the non-transitive case. Denote by $X_{i}$ the (finitely many) $B$-orbits of $X$. Consider an action of $G$ as in the statement of the proposition. Then, for some $i, T^{A^{\left(X_{i}\right)}} \neq \emptyset$. By the transitive case, $A^{\left(X_{i}\right)}$ preserves a unique line $\mathcal{L} \subset T$, on which it acts non-trivially and by translations. Set $Y=X-X_{i}$. Then $A^{(Y)}$ centralizes $A^{\left(X_{i}\right)}$, so preserves $\mathcal{L}$. Moreover, $A^{(Y)}$ also preserves the orientation of $\mathcal{L}$ (otherwise as in the transitive case, it contains a point with a unique fixed point, which is then fixed by a hyperbolic element in $A^{\left(X_{i}\right)}$, a contradiction).

Proof of Theorem 1.1. Under the conditions of the theorem, suppose that the permutational wreath product $G:=A 2_{X} B$ has Property (FA). Then clearly $B$, being a quotient of $G$, has Property (FA). Moreover, $A$ cannot be written as a properly increasing union of a sequence of subgroups $\left(A_{n}\right)$, since otherwise (using $X \neq \emptyset$ ) $G$ would be the increasing union of its subgroups $A_{n} 2_{X} B$ and would fail to have Property (FA). Moreover, since $X$ has no one-element orbit, the abelianisation of $G$ 
is given by $G^{\mathrm{ab}}=\left(A^{\mathrm{ab}}\right)^{Y} \times B^{\mathrm{ab}}$, where $Y$ denotes the orbit set $B \backslash X$. Hence the abelianisation of $A$ is also finite.

Conversely, suppose that $B$ has property (FA) and the group $A$, which has finite abelianisation, cannot be written as a properly increasing union of its subgroups. Let $G$ act without inversions on a tree $T$. To verify that $G$ has property (FA) we need to prove that $T^{G}$ is non-empty.

Write $W=A^{(X)}$. Suppose that $T^{W} \neq \emptyset$. Then this is a nonempty subtree the action on which factors through $B$. So by Property (FA) for $B$, we obtain $T^{G} \neq \emptyset$.

Otherwise, $T^{W}=\emptyset ; T^{B} \neq \emptyset$ by Property (FA) for $G$, and the action of $A_{x}$ is not parabolic by Lemma 2.2. So Proposition 2.3 implies that there is a non-trivial homomorphism from $A^{(X)}$ to $\mathbf{Z}$. This is impossible since $\operatorname{Hom}\left(A^{(X)}, \mathbf{Z}\right)=\operatorname{Hom}(A, \mathbf{Z})^{X}=\{0\}$.

Lemma 2.4 (I.6.5.10 in [16]). Let $T_{1}, \ldots, T_{m}(m \geq 2)$ be subtrees of a tree $T$. If the $T_{i}$ have pairwise nonempty intersection, then their intersection is non-empty.

Proof of Proposition 1.3. We restrict ourselves to the case when $X$ is $B$-transitive; the reader can easily deduce the general case.

Fix a basepoint $o$ in $X$ and let $C \subset B$ be its stabilizer. Fix finite generating subsets $S_{A}, S_{B}$ of $A$ and $B$. Consider the group $K$ obtained from the free product $A * B$ by adding the relators

(1) $[c, a]\left(c \in C \cap S_{B}, a \in S_{A}\right)$;

(2) $\left[b a b^{-1}, a^{\prime}\right]\left(b \in S_{B}-C, a, a^{\prime} \in S_{A}\right)$.

In $K$, for $b \in B$ write $A_{b}=b A b^{-1}$. Let $K$ act on a tree $T$. By Lemma 2.4, it is enough to check that any pair of generators of $K$ has a common fixed point. Since $B$ has Property (FA), every pair in $S_{B}$ has a common fixed point. The proof now proceeds along the same lines as that of Proposition 2.3

- Suppose $T^{A_{1}} \neq \emptyset$. Thus, every pair of generators in $S_{A}$ has a common fixed point. It remains to show that any pair $(a, s) \in$ $S_{A} \times S_{B}$ has a common fixed point. Let us first check that $T^{A_{1}} \cap T^{A_{s}} \neq \emptyset$.

- If $s \in C$, then, thanks to relators of type (1), we have $[s, A]=\{1\}$, hence $A_{s}=A_{1}$, so this is clear;

- If $s \notin C$, then the relators of type (2) assure us that $\left[A_{1}, A_{s}\right]=\{1\}$. Hence by Lemma 2.1, $A_{1}$ and $A_{s}$ have a common fixed point.

As in the proof of Theorem 1.1, we denote the point in $T^{A_{b}}$ closest to $T^{B}$ as $u^{b}$. Now, $u^{1}=s \cdot u^{1}=u^{s}$ for all $s \in S_{B}$, so 
this is a fixed point for $B$. Thus, $a$ and $s$ have a common fixed point.

- Suppose $T^{A_{1}}=\emptyset$. Observe that $S_{B}-C$ is non-empty else $X$ would have contained only one element. Pick $b \in S_{B}-C$. The subgroups $A_{1}$ and $A_{b}$ contain elements $a, a^{\prime}$ respectively whose actions on $T$ are hyperbolic. The axis $\mathcal{L}$ of $a^{\prime}$ is stabilised by $A_{1}$. Moreover every element of $A_{1}$ preserves the orientation of $\mathcal{L}$ for otherwise, its unique fixed point is a fixed point for $a^{\prime}$. Thus the action of $A_{1}$ on $\mathcal{L}$ is by translations. Since $\operatorname{Hom}(A, \mathbf{Z})=\{0\}$, we deduce that $A_{1}$ acts trivially, contradicting the existence of $a$.

\section{Hereditary Property (FA)}

We need the following classical lemma.

Lemma 3.1. Let $G_{0}$ be a group and let $\left(N_{k}\right)_{k}$ be a non-decreasing sequence of normal subgroups of $G_{0}$. Set $G=G_{0} / N$, where $N=\bigcup N_{k}$ and $G_{k}=G_{0} / N_{k}$. Let $H$ be a finitely presented group. Then every homomorphism $f: H \rightarrow G$ lifts to a homomorphism $f_{k}: H \rightarrow G_{k}$ for some $k$. If in addition, $f$ is surjective and $G_{0}$ is finitely generated, then $f_{k}$ can be chosen to be surjective.

Proof. It is a standard result that a group $H$ is finitely presented (if and) only if the functor $\operatorname{Hom}(H,-)$ commutes with inductive limits (see [1], Example 1.2(5) and Corollary 3.13, which reach far beyond the realm of groups). Since $G=\underline{\lim } G_{k}$, this proves the existence of an $f_{k}$.

Suppose now that $f$ is surjective and $G_{0}$ has a finite generating subset $S$. For every $s \in S$, the image of $s$ into $G$ belongs to the image of $f$; thus there exists $g_{s}$ in the kernel of $G_{k} \rightarrow G$ such that $s g_{s}$ belongs to $f_{k}(H)$. Since $S$ is finite, there exists $\ell \geq k$ such that $g_{s}=1$ in $G_{\ell}$ for all $s \in S$. Thus the composite map $f_{\ell}: H \rightarrow G_{k} \rightarrow G_{\ell} \operatorname{lifts} f$ and is surjective.

Proof of Theorem 1.5. If $F$ is a finite subset of $B-\{1\}$, define $\Gamma(A, B, F)$ as the quotient of $A * B$ by the "relators" $\left[A, u A u^{-1}\right]$ for $u \leq B$. Let $\left(u_{k}\right)_{k \geq 1}$ be an enumeration of $B-\{1\}$ and define, for $k \leq \infty$

$$
G_{k}=\Gamma\left(A, B,\left\{u_{i}: i \leq k\right\}\right) .
$$

Note that $G_{\infty}=A$ < $B$.

The group $\Gamma(A, B, F)$ has a natural semidirect product decomposition $M \rtimes B$, where $M=M(A, B, F)$ is a "graph product" (see [5, Section 2]). This means that $M$ is the free product of copies $A_{b}$ of 
$A$ indexed by $b \in B$ and subject to the relations $\left[A_{b}, A_{b s}\right]=1$ for all $s \in F$, and $u \in B$ shifts $A_{b}$ to $A_{u b}$.

Let $H$ be a finitely presented group having $G$ as a quotient. Then $H$ has $G_{k}$ as a quotient for some $k$. So we only have to prove that $G_{k}$ has a finite index subgroup mapping onto a free group.

We prove the following general statement. Let $A$ and $B$ be groups such that $A$ has at least one non-trivial finite quotient $A_{1}$ and $B$ is residually finite. Suppose $F$ is a symmetric subset of $B-\{1\}$ such that there exists $c, d \in B$ satisfying $\left\{c, d, c^{-1} d\right\} \cap(F \cup\{1\})=\emptyset$. Then $\Gamma(A, B, F)$ has a finite index subgroup mapping onto a non-abelian free group.

First observe that $\Gamma(A, B, F)$ maps onto $\Gamma\left(A_{1}, B, F\right)$, so replacing $A$ by $A_{1}$ if necessary, we can assume that $A$ is finite and non-trivial. Let $N$ be a normal subgroup of finite index in $B$ such that $F \cup\{1, c, d\}$ is mapped injectively into $B^{\prime}=B / N$. Since the image of $F$ in $B^{\prime}$ (still written $F)$ is nontrivial, the group $\Gamma\left(A, B^{\prime}, F\right)$ is well-defined; this is a quotient of $\Gamma(A, B, F)$. Using the graph product description given above, we write $\Gamma\left(A, B^{\prime}, F\right)=M \rtimes B^{\prime}$, with $M=M\left(A, B^{\prime}, F\right)$. So $M$ is a finite index subgroup of $\Gamma\left(A, B^{\prime}, F\right)$. Taking the quotient of $M$ by the normal subgroup generated by all $A_{b}$ for $b \neq\{1, c, d\}$, we see that all relators become trivial and therefore we obtain the free product $A_{1} * A_{c} * A_{d}$. The latter group has a non-abelian free subgroup of finite index.

Proposition 3.2. Consider the short exact sequence of groups:

$$
1 \rightarrow A \rightarrow G \rightarrow B \rightarrow 1
$$

Assume that $A$ does not contain any nonabelian free subgroup, and that $G$ is finitely generated (or more generally, is not the union of a properly increasing sequence of subgroups) and does not map onto the integers or the infinite dihedral group. Then, $G$ has Property (FA) if and only if $B$ has Property (FA).

Proof. If $G$ is a group, define $\mathrm{NF}(G)$ to be the largest normal subgroup of $G$ without nonabelian free subgroups. The $\operatorname{subgroup~} \mathrm{NF}(G)$ is always well-defined. Indeed, let $N_{1}$ and $N_{2}$ be normal subgroups of $G$ with no nonabelian free subgroups. Then $N_{1} N_{2}$ is also normal and the second isomorphism theorem implies that $N_{1} N_{2}$ cannot contain a non-abelian free subgroup.

The "only if" part of the proposition is clear. Conversely, suppose that $G$ fails to have Property (FA). Then $G$ splits as a non-trivial amalgam $H *_{K} L$. If the amalgam were degenerate ( $K$ has index two in both $H$ and $L$ ), then $G$ would map onto the infinite dihedral group. 
Therefore, we can apply [6, Proposition 7], which says in particular that $\operatorname{NF}(G)$ is contained in $K$. Since $A$ is by definition contained in $\mathrm{NF}(G)$, this shows that $G / A \cong B$ splits as a non-trivial amalgam $(H / A) *_{K / A} L / A$, and therefore fails to have Property (FA).

Proof of Theorem 1.6. The fact that the first condition implies the second one is as straightforward as the analogous implication for Theorem 1.1, so we do not repeat the argument.

So assume that $A$ has finite abelianisation and $B$ has hereditary Property (FA).

We first prove the implication when $A$ has trivial abelianisation, as the proof is then easier. In this case, by Gruenberg [12] every finite index subgroup of $G$ contains the normal subgroup $A^{(B)}$ and is therefore of the form $A^{(B)} \rtimes C$ where $C$ has finite index in $B$; since $B$ is supposed to be infinite, $C$ is non-trivial. This group $A^{(B)} \rtimes C$ is a permutational wreath product (with a non-transitive free action), so Theorem 1.1 applies.

Before passing to the general case, we need to consider the special case when $A$ is abelian (and thus finite). Every finite index subgroup $H$ of $G$ then is an extension of groups such that the kernel $K$ is torsion (and abelian) and the quotient is a finite index subgroup of $B$. We claim that $H$ has no quotient $Q$ isomorphic to the group of integers or the infinite dihedral group. Suppose on the contrary that $Q \cong \mathbb{Z}$ or $D_{\infty}$. Since $Q$ has no non-trivial torsion normal subgroup, the image of $K$ into $Q$ is trivial, so $Q$ is a quotient of $B$. But this is absurd since $B$ has Property (FA) by hypothesis. So we can apply Proposition 3.2 to deduce that $H$ has Property (FA).

Suppose now, in general, that the derived subgroup $D$ of $A$ has finite index in $A$, and that $H$ has finite index in $G$; let $H$ act on a tree $T$. Then Gruenberg [12 implies that $H$ contains $D^{(B)}$. We claim that $T^{\prime}=T^{D^{(B)}} \neq \emptyset$. Suppose $D^{(B)}$ has no global fixed point. Set $C=B \cap H$. The group $G^{\prime}=D^{(B)} \rtimes C$ is a permutational wreath product; $B$ being a free $C$-set. Since $B$ is infinite, $C$ is non-trivial, so Proposition 2.3 applies. Therefore, $D^{(B)}$ preserves a unique line, on which it acts by non-trivial translations. Since $D^{(B)}$ is normal, this line is preserved by $G^{\prime}$. The action on this line is given by a homomorphism from $G^{\prime}$ to the infinite dihedral group. Since $D_{\infty}$ is residually finite, Gruenberg's theorem imples that the homomorphism is trivial on $D^{(B)}$. This is impossible and so $T^{\prime}=T^{D^{(B)}} \neq \emptyset$.

Finally, since $D^{(B)}$ is normal in $H$, we know that $T^{\prime}$ is $H$-invariant and that the action of $H$ on $T^{\prime}$ factors through $H / D^{(B)}$, which is a subgroup of finite index of $(A / D)$ 乙 $B$. By the special case when the 
base group is abelian, we deduce that there is a fixed point. This proves that $H$ has Property (FA).

\section{REFERENCES}

[1] J. Adámek, J. Rosický. Locally presentable and accessible categories. London Math. Soc. Lectures Notes Series 189, Cambridge Univ. Press, 1994.

[2] G. Baumslag, Wreath products and finitely presented groups, Math. Z. 75, 22-28 (1961).

[3] Y. de Cornulier and L. Bartholdi, Infinite groups with large balls of torsion elements and small entropy, Archiv der Mathematik 82(2), 104-112, 2006.

[4] Y. de Cornulier, A note on quotients of word hyperbolic groups with Property (T) (2005), arXiv:0504.5193v3.

[5] Y. de Cornulier, Finitely presented wreath products and double coset decompositions. Geom. Dedicata 122 (2006), 89-108.

[6] Y. Cornulier. Infinite conjugacy classes in groups acting on trees. Groups Geom. Dyn. 3(2) (2009) 267-277.

[7] B. Bekka, P. de la Harpe, A. Valette, Kazhdan's Property (T), Cambridge University Press, 2007.

[8] C. Champetier, V. Guirardel. Limit groups as limits of free groups: compactifying the set of free groups. Israel J. Math. 146, 1-76, 2005.

[9] M. Culler and J.W. Morgan, Group Actions on R-trees, Proc. London Math. Soc (3) 55 (1987) 571-604.

[10] R. I. Grigorchuk and Pierre de la Harpe, Limit behaviour of exponential growth rates for finitely generated groups, Monogr. Enseign. Math. 38, 351370 (2001).

[11] M. Gromov, Random Walk in Random Groups, Geom. Funct. Anal. 13. (2003), No. 1, 73-146.

[12] K. W. Gruenberg. Residual properties of infinite soluble groups. Proc. London Math. Soc. (3) 7 (1957) 29-62.

[13] P. de la Harpe, Topics in Geometric Group Theory, Univ. of Chicago Press, 2000.

[14] S. Koppelberg, J. Tits. Une propriété des produits directs infinis de groupes finis isomorphes. C. R. Acad. Sci. Paris, Ser. A 279 (1974), 583-585.

[15] G. Margulis, On the decomposition of discrete subgroups into amalgams. Selected translations, Selecta Math. Soviet. 1 (1981) 197-213.

[16] J-P. Serre, Trees, Springer Monographs in Mathematics, 1980.

[17] P.B. Shalen, Dendrology of groups: an introduction, Essays in group theory, 265319, Math. Sci. Res. Inst. Publ. 8, Springer, 1987.

[18] Y. Shalom, Rigidity of commensurators and irreducible lattices. Inv. Math., 141:1-54, 2000.

[19] Y. Stalder, Fixed point properties in the space of marked groups, in 'Limits of graphs in group theory and computer science', p. 171-182, Proc. Special semester at Centre Bernoulli (Lausanne), Jan-June 2007, G. Arzhantseva and A. Valette editors, Presses Polytechniques et Universitaires Romandes; arXiv:0803.2592.

[20] J. Tits, Sur le groupe des automorphismes d'un arbre. In "Essays on topology and related topics (Mémoires dédiés à Georges de Rham), 188-211. Springer, New York, 1970. 
[21] Y. Watatani, Property (T) of Kazhdan implies Property (FA) of Serre. Math. Japonica, 27:97-103, 1982.

IRMar, Campus de Beaulieu, 35042 Rennes CEDEX, France

E-mail address: yves.decornulier@univ-rennes1.fr

School of Mathematics, University of Southampton, Southampton SO17 1BJ, UK

E-mail address: A.Kar@soton.ac.uk 